[14] Marohand eit. in Martiny, 1. c. S. 243.

[15] Mantivy B., Die Milch, ihr Wesen und ihre Ververtung, Danzig, A. W. Kafemann, 1871.

[16] Ménard, Extrait d'une lettre de Ménard, Annal. de l'agric. franc., 1857, S. 288.

[17] Möвгоs P. J., Uber die Wirkung der Kastration, Beitrag zur Lehre von den Geschlechtsunterschieden, Heft 3/4, C. Marhold, Leipzig 1903, S. 37.

[18] MüNCHBerg F., Milchw. Forschungen, 3, 404, 1926.

[19[ NuEsch, Berl., Tierarztl. Wochenschr. 1910, No 47.

[20] Retzios, Om Kastration af Kor, Stockholm 1835, S. 6, cit. in Martiny, 1. c. S. 241.

[21] Rossmeiser J., Biochem. Zeitschr., 16, 164, 1909.

[22] Spann, Landw. Fachpresse f. d. Tschechoslowakei, 8, 1, 1930.

[23] WINN, Recueil de médec. vétér. prat, 1834 cit. in Martiny, 1. e. S. 241.

[24a] ZARIBNICKY F., Milchw. Forschungen 3, 404, 1926.

[24b] ZaRIBNIoKY F., Wiener, Tierärztl. Monatschrift, 14, 450, 1927.

\title{
OBSERVATIONS PRATIQUES SUR LE CALCUL DU RENDEMENT EN BEURRERIE
}

\author{
par M. E. VAILLANT, \\ Ingénieur agronome
}

(Fin)

\section{DISCUSSION ET INTERPRÉTATION DU CALCUL DE RENDEMENT.}

Afin de nous permettre de tirer de ces ealculs tous les enseignements qu'ils comportent, nous reproduisons ci-dessous la copie d'une page du livre de fabrication d'une usine où cette méthode est a ppliquée. Armé de ce document, nous allons l'étudier en détail et tirer, de tous les chiffres qui y sont consignés, les commentaires, constatations et critiques qu'ils a ppellent.

Avant de prendre contact avec ce tableau, nous croyons utile de situer exactement l'usine et le travail qui s'y fait.

La laiterie qui nous intéresse est installée dans un important centre d'élevage de vaches hollandaises (ce qui explique la faible teneur en matière grasse du lait à cette époque de l'année). Cette laiterie, qui ramasse en moyenne 10.000 litres par jour en hiver et 18.000 litres en été, livre à la consommation une moyenne journalière assez régulière de 5.500 à 6.000 litres. Lo reste est travaillé à l'usine pour être écrémé. Le beurre obtenu est en partie mis en pains et vendu à la ville voisine, l'autre partie est expédiée en mottes de $10 \mathrm{~kg}$. ou en caisses de $25 \mathrm{~kg}$.

Lo lait écrémé, a près distribution aux producteurs de la quantité 


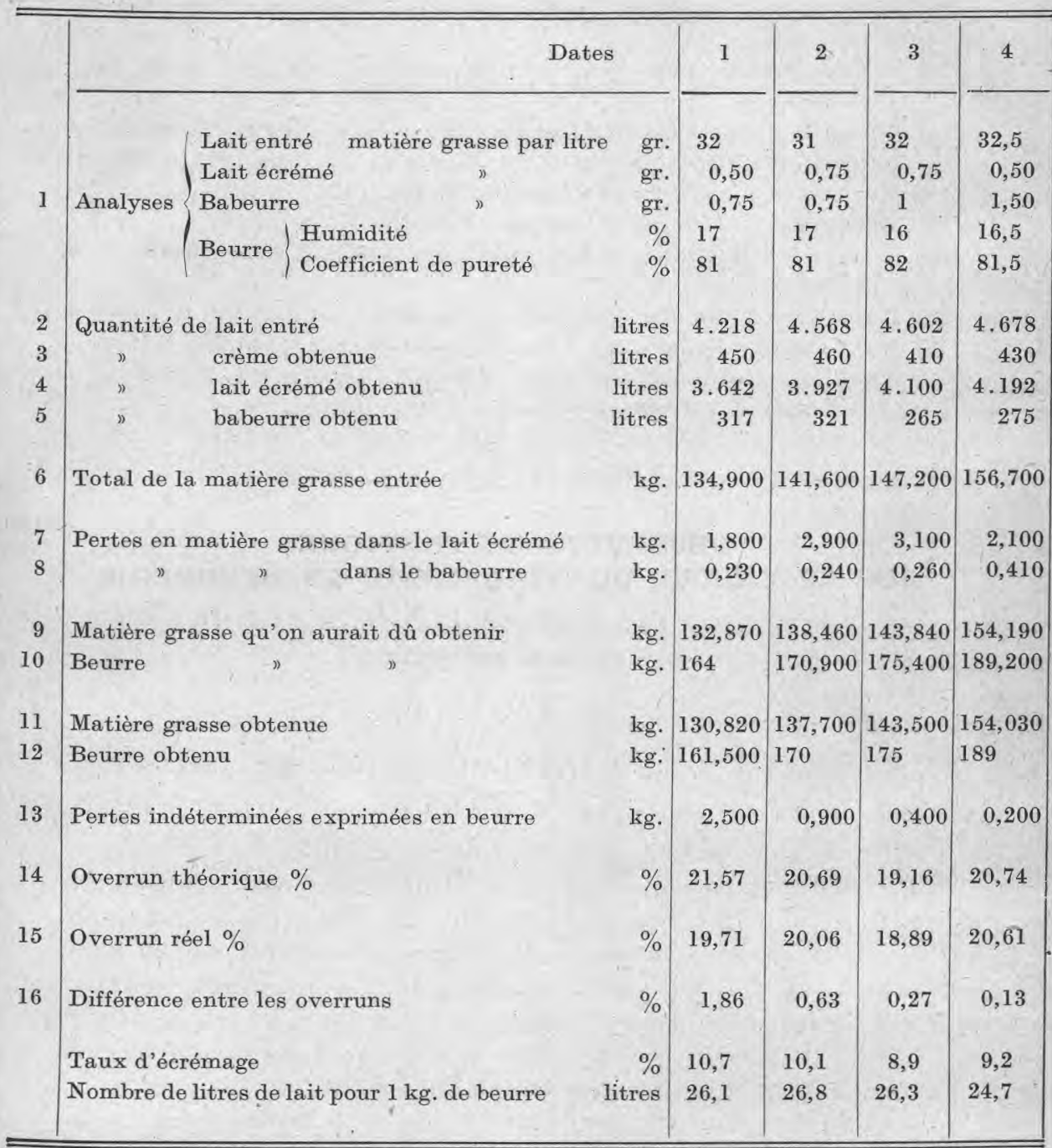

qu'ils demandent, est entièrement transformé en caséine lactique qui est séchée sur place. Enfin, le babeurre est livré à une porcherie des environs.

La réception du lait se fait " au litre " par simple jaugeage. Le lait n'est pas contrôlé à l'usine, mais la Société possède 2 contrôleurs qui, en permanence, accompagnent les ramasseurs et prélèvent les échantillons au chargement, devant le producteur. Lorsqu'une anomalie 


\begin{tabular}{|c|c|c|c|c|c|c|c|c|c|c|}
\hline 5 & 6 & 7 & 8 & 9 & 10 & 11 & 12 & 13 & 14 & 15 \\
\hline 34 & 33 & 32 & 33 & 33 & 31,5 & 32 & 33 & 33 & 33 & 31,5 \\
\hline 0,75 & 0,75 & 0,50 & 1 & 0,75 & 2 & 0,50 & 1 & 0,50 & 0,75 & 0,50 \\
\hline 1 & 2 & 2 & 3,5 & 2 & 3,5 & 5 & 2 & 2 & 2 & 3 \\
\hline 17,5 & 17,5 & 17 & 15,5 & 14,5 & 17 & 16 & 16 & 15,5 & 17 & 18 \\
\hline 80,5 & 80,5 & 81 & 82,5 & 83,5 & 81 & 82 & 82 & 82,5 & 81 & 80 \\
\hline 4.591 & 4.708 & 4.703 & 4.853 & 4.969 & 4.954 & 4.924 & 4.844 & 4.825 & 4.618 & 4.800 \\
\hline 460 & 450 & 450 & 440 & 440 & 450 & 460 & 450 & 450 & 410 & 500 \\
\hline 4.000 & 4.200 & 4.190 & 4.377 & 4.422 & 4.451 & 4.380 & 4.270 & 4.300 & 4.200 & 4.240 \\
\hline 307 & 298 & 302 & 284 & 279 & 253 & 305 & 294 & 293 & 265 & 351 \\
\hline 156,100 & 156,360 & 150,500 & 160,140 & 163,970 & 165,900 & 157,500 & 159,850 & 159,220 & 147,770 & 151,200 \\
\hline 3,000 & 3,150 & 2,100 & 4,300 & 3,300 & 8,900 & 2,200 & 4,200 & 2,150 & 3,150 & 2,100 \\
\hline 0,300 & 0,580 & 0,600 & 0,990 & 0,560 & 0,870 & 1,530 & 0,590 & 0,580 & 0,530 & 1,050 \\
\hline 152,800 & 152,630 & 147,800 & 154,850 & 160,110 & 156,130 & 153,770 & 155,060 & 156,490 & 144,090 & 148,050 \\
\hline 189,800 & 189,600 & 182,400 & 187,700 & 191,600 & 192,800 & 187,500 & 189 & 189,700 & 177,900 & 185 \\
\hline 152,550 & 149,730 & 147,420 & 153,450 & 154,480 & 154,710 & 152,520 & 154,160 & 155,930 & 142,160 & 147,400 \\
\hline 189,500 & 186 & 182 & 186 & 185 & 191 & 186 & 188 & 189 & 175,500 & 184,250 \\
\hline 0,300 & 3,600 & 0,400 & 1,700 & 6,600 & 1,800 & 1,500 & 1,000 & 0,700 & 2,400 & 0,750 \\
\hline 21,59 & 21,26 & 21,19 & 17,21 & 16,85 & 16,21 & 19,04 & 18,23 & 19,14 & 20,39 & 22,35 \\
\hline 21,39 & 18,95 & 20,93 & 16,14 & 12,82 & 15,13 & 18,09 & 17,61 & 18,70 & 18,76 & 21,85 \\
\hline 0,20 & 2,31 & 0,26 & 1,07 & 4,03 & 1,08 & 0,95 & 0,62 & 0,44 & 1,63 & 0,50 \\
\hline 10 & 9,6 & 9,6 & 9,07 & 8,85 & 9,07 & 9,3 & 9,28 & 9,3 & 8,87 & 10,4 \\
\hline 24,2 & 25,3 & 25,8 & 26,1 & 26,8 & 25,9 & 26,5 & 25,6 & 25,5 & 26,3 & 26 \\
\hline
\end{tabular}

est reconnue, ces mêmes contrôleurs vont faire des prélèvements de comparaison à l'étable.

L'écrémage est fait à un taux voisin de $10 \%$, plutôt un peu moins. La crème est pasteurisée à $80^{\circ}-85^{\circ}$, refroidie sur un réfrigérant à 2 eaux, pompée vers les cuves de maturation où elle reçoit du levain de ferments danois.

Le barattage enfin s'effectue dans une baratte-malaxeur et le 
beurre est emmagasiné dans l'antichambre d'une installation frigorifique où, selon les saisons, on fait varier la température qu'on maintient aussi fraîche que possible (le beurre de cette région ayant une tendance marquée à se tenir un peu mou).

Ajoutons pour être complet que l'installation frigorifique fournit la glace nécessaire aux opérations de la beurrerie en saison chaude et qu'en dehors de la machine à vapeur qui entraîne le matériel de l'usine, la force motrice actionnant l'installation frigorifique est constituée par un moteur Diesel de $25 \mathrm{CV}$. sur lequel est attelée également une génératrice électrique fournissant la force nécessaire au fonctionnement de la caséinerie.

Ceci posé, revenons à l'étude du travail de cette usine, étude qui nous est grandement facilitée par les observations très judicieuses ajoutées chaque jour au tableau de rendement par le Directeur, et qui portent surtout sur les événements et constatations de la journée a yant une répercussion possible et même probable sur les résultats. (Nous utiliserons ces observations dans les explications qui vont suivre.)

Rósumons d'un mot l'ensemble des manipulations à effectuer en cours de journée et qui nous sont indispensables :

$1^{0}$ Recueillir de la façon la plus présise un échantillon moyen de lait entré, de lait écrémé et de babeurre obtenu.

Les analyser en ayant bien soin d'interpréter les résultats des analyses avec la plus grande approximation possible, e'est de toute première importance ;

$2^{\circ}$ Mesurer a vec précision le lait entré et la erème obtenue. Nous avons vu que ces deux résultats nous permettent d'obtenir très facilement les volumes du lait écrémé et du babeurre ;

$3^{\circ}$ Peser très consciencieusement le beurre obtenu, titrer avec soin l'humidité qui nous donne immédiatement le coefficient de pureté du beurre.

Il nous suffira maintenant d'effectuer les calculs.

\section{DisOUSSION DE L'OVERRUN THÉORIQUE.}

Tous les résultats que nous avons obtenus ont tendu à nous procurer l'overrun.

Par définition, ainsi que nous l'avons indiqué au début de ce travail, l'overrun théorique est la différence entre le poids de matière grasse entrée et le poids de beurre qu'on aurait dû obtenir.

Si le travail était parfait, si toute la matière grasse contenue dans le lait reçu à l'usine avait été transformée intégralement en beurre, sans perte d'aucune sorte, cet overrun représenterait strictement le total des impuretés du beurre.

Si nous admettons que le beurre contient $16 \%$ d'eau et $2 \%$ d'im- 
puretés diverses, $82 \mathrm{~kg}$. de matière grasse entrée auront dû donner $100 \mathrm{k}$. de beurre; l'overrun dans ce cas est :

$$
100-82=18 \text {. }
$$

Comme on l'exprime en $\%$ de la matière grasse entrée, cet overrun théorique absolu \% serait :

$$
\frac{18 \times 100}{82}=21,9 \%
$$

Mais nous savons, par expérience, que, dans la pratique, une certaine proportion de matière grassè se perd, à l'écrémage, dans le lait écrémé, et au barattage, dans le ba beurre ; nous pou vons donc affirmer, $q u$ 'à coefficient de pureté égal, l'overrun théorique \% trouvé dans une beurrerie sera toujours inférieur à 21,9 . Il s'écartera d'autant plus de ce chiffre que les pertes en cours de fabrication seront plus élevées.

$$
* * *
$$

INFIUENCE DES PERTES CHIFFRÉES SUR L'OVERRUN THÉORIQUE.

Il est possible d'apprécier par un simple calcul quelle peut être l'influence de ces pertes sur l'overrun théorique.

Prenons une usine écrémant 10.000 litres de lait et tirant 1.0001 . de crème, elle aura donc recueilli 9.000 litres de lait écrémé. Supposons que le lait entré titre en moyenne 32 gr. de matière grasse par litre, l'usine aura done reçu $320 \mathrm{~kg}$. de matière grasse. Si nous admettons momentanément que la pureté de beurre obtenu est de $82 \%$, ces $320 \mathrm{~kg}$. de matière grasse devraient donner :

$$
\frac{320 \times 100}{82}=390 \mathrm{~kg} \text {. de beurre, }
$$

et l'overrun théorique absolu \% demeure bien :

$$
\frac{(390-320) \times 100}{320}=21,9 \% \text {. }
$$

Ceci en ne supposant aucune perte dans le lait écrémé ni dans le babeurre.

Cherehons quelle sera l'influence d'une perte de 0 gr. 1 par litre de lait écrémé.

Pour 9.000 litres elle sera de 0 k. 900.

On aura donc baratté $320-0,900=319,100$ de matière grasse qui devra donner, à la pureté de $82 \%$ :

$$
\frac{319.100 \times 100}{82}=389 \text { k. } 140 \text { de beurre, }
$$


L'overrun théorique devient dans ce cas :

$$
\frac{(389,140-320) \times 100}{320}=21,60 \% \text {. }
$$

Donc 0 gr. 1 de matière perdue par litre de lait écrémé fait baisser le pourcentage d'overrun théorique $\%$ de 0,30 .

Un calcul identique montrerait que $1 \mathrm{gr}$. de matière grasse perdue dans le babeurre fait baisser le pourcentage d'overrun théorique $\%$ de 0,25 .

Si nous prenons eomme contrôle de ces conclusions les chiffres de la journée du 3 décembre par exemple, oü le beurre a une pureté de 82 , nous pouvons dire immédiatement que :

$1^{\circ}$ l'overrun théorique a bsolu est abaissé par la perte dans le lait écrémé (qui titre 0,75 par litre) de :

$$
0,34 \times 7,5=2,55 \text {; }
$$

$2^{\circ}$ il est également abaissé par la perte dans le babeurre (qui titre 1 gr. par litre) de 0,25 :

$$
\text { soit au total } 2,55+0,25=2,80 \text {; }
$$

il doit donc être de :

$$
21,9-2,80=19,10 \%
$$

Chiffre qui se rapporte bien à celui obtenu par le calcul direct qui est $19,16 \%$.

(La petite différence qui existe entre ces deux chiffres est principalement due à ce que, dans le tableau de rendement que nous a vons -pris comme exemple, le volume de lait écrémé a été mesuré et se trouve de ce fait inférieur au volume obtenu par différence entre le lait entré et la crème récoltée.)

\section{$*^{*} *$ \\ INFLUENCE DU TAUX D'HUMIDITÉ DU BEURRE SUR L'OVERRUN THÉORIQUE}

Nous avons jusqu'ici posé comme base de nos calculs, afin de les rendre absolument comparatifs, une fixité absolue du coefficient de pureté $(82 \%)$, parce que nous avons admis que l'humidité du beurre fabriqué était de $16 \%$. En pratique, rien n'est moins fixe que cette teneur en eau et il est rare de trouver une usine où la fabrication montre une régularité aussi absolue, notamment dans certaines périodes de l'année.

En été, des chaleurs excessives viennent gêner le travail, en hiver, ce sont des gelées brusques et violentes qui amènent des perturbations dans le barattage. Si le beurrier doit a voir la clairvoyance néces- 
saire pour en éviter l'influence, il n'y parvint pas toujours immédiatement et quelquefois, lorsque les conditions de milieu ou les possibilités matérielles ne le favorisent pas, il éprouve à ce point de vue de grosses difficultés.

C'est ainsi que nous constatons dans l'usine qui nous intéresse une augmentation croissante de l'humidité du 4 au 6 décembre, puis un essai de diminution le 7 , suivi de 2 jours oü cette humidité a baissé sensiblement, et même au-dessous du chiffre couramment admis (ce qui est désavantageux pour l'exploitation), il essaie de remonter ce taux le 10 , le stabilise le 11 , pour retomber le 14 , et surtout le 15 , dans un fort excès d'humidité.

Evidemment le coefficient de pureté suit en sens inverse les mêmes péripéties. Il augmente quand l'humidité diminue et réciproquement.

Voyons quelle sera l'influence de ces variations sur l'overrun théorique :

Si nous reprenons un calcul semblable à celui qui nous a servi à établir l'influence des pertes sur l'overrun théorique, nous pouvons constater que :

Si les $320 \mathrm{~kg}$. de matière grasse avaient donné du beurre à $17 \%$ d'humidité, done ayant un coefficient de pureté de $81 \%$, l'overrun théorique absolu \% aurait augmenté de 1,55, tandis que si le beurre obtenu avait eu une humidité de $15 \%$, done un coefficient de pureté de $88 \%$, l'overrun \% aurait diminué de 1,45.

Nous en concluons :

1. Que l'augmentation de $1 \%$ de l'humidité augmente l'overrun de 1,55 et que la diminution de $1 \%$ de 1 'humidité le diminue de 1,45 ;

$2^{\circ}$ Que l'augmentation et la diminution de l'humidité n'ont pas une influence égale sur l'overrun ;

$3^{\circ}$ Que cette influence est très importante.

\section{** $*$ \\ INTERPRETATION FINALE DU CHIFFRE DE L'OVERRUN THÉORIQUE.}

Ces constatations nous prouvent done que l'obtention d'un beurre trop humide peut voiler complètement l'influence des pertes en cours de fabrication et créer ainsi, à la simple lecture de l'overrun théorique, une erreur grossière d'interprétation.

Nous nous empressons de dire qu'aucun industriel beurrier ne se laissera prendre, car la crainte des représailles du Service des Fraudes lui donne la sagesse de porter tout d'abord son attention sur le taux d'humidité de son beurre, et il peut immédiatement rétablir son chiffre d'overrun et le commenter utilement en faisant abstraction, par 
un simple calcul mental, de l'influence de ce taux d'humidité sur le chiffre lu.

Prenons comme exemple la journée du 15 décembre.

Au premier coup d'œil sur les analyses, nous voyons que le beurre a une humidité vraiment anormale (18\%).

Regardons l'overrun théorique, il est supérieur au chiffre de 21,9 que nous avions pris comme base, puisqu'il fait $22,35 \%$.

Rectifions-le. Il y a $2 \%$ d'humidité en trop correspondant donc à une augmentation de l'overrun de $1,55 \times 2=3,10$. Cet overrun, s'il était normal devrait done être de $22,35-3,10=19,25 \%$.

Vérifions maintenant si ce chiffre se rapporte bien aux pertes enregistrées.

Le lait écrémé titre 0,50 , donc a dû opérer une diminution de l'overrun de :

$$
0,34 \times 5=1,70 .
$$

Le babeurre titre 3 , soit une diminution de :

$$
0,25 \times 3=0,75,
$$

done diminution totale sur l'overrun théorique absolu de :

$$
1,70+0,75=2,45 \text {, }
$$

ce qui donne $21,9-2,45=19,45$, chiffre très voisin du chiffre rectifié $(19,25)$ trouvé ci-dessus.

En résumé, l'overrun théorique absolu est de $21,9 \%$ de la matière grasse entrée. Il n'est jamais atteint, mais se trouve toujours diminué par les pertes inévitables résultant de la matière grasse entraînée dans le lait écrémé et le babeurre; il peut être accidentellement augmenté par une humidité anormale du beurre, augmentation qui, à première vue, peut contrebalancer la diminution due aux pertes et même amener l'overrun au-dessus du chiffre théorique de 21,9. Un simple examen du dosage de l'humidité suffit à rétablir les chiffres à leur valeur réelle.

\section{DisCUSSION DE L'OVERRUN RÉEL.}

Contrairement à l'overrun théorique qui ne repose que sur le beurre qu'on aurait d̂̂ obtenir, l'overrun réel repose sur le beurre qu'on a effectivement obtenu. Il est, en somme, l'image réelle du tra vail de l'usine; il n'est plus le résultat d'un ealcul basé sur des chiffres théoriques, où les accidents du travail n'ont rien à voir. L'overrun réel repose sur des résultats tangibles, il est d'une sincérité brutale, indiscutable.

Nous ne pouvons, à son sujet, entreprendre des calculs où la supposition règne en maître; nous sommes obligés de nous servir des seuls résultats fournis par le personnel de l'usine, résultats qui sont eux- 
mêmes le fruit de dosage, de mesurages et de pesées réels effectués avec conscience au cours du travail .

Nous en profiterons pour renouveler encore une fois ici le conseil extrêmement important de veiller à obtenir, pour toutes ces opérations d'analyses ou de pesées et de mesurages, la précision la plus grande possible. Sans cette eondition, tous les calculs sont entachés d'erreurs grossières qui en rendent les résultats faux, inutiles et fantaisistes.

Nous savons que l'overrun réel est la différence entre la matière grasse totale entrée et le beurre réellement obtenu.

Cet overrun, comme le précédent, s'exprime toujours en $\%$ de la matière grasse totale entrée.

Rien, dans ce calcul, n'est laissé ni au hasard, ni à l'empirisme. Toutes les données sont contrôlables. La matière grasse entrée nous est donnée par le nombre de litres de lait reconnu à l'entrée de l'usine et par l'analyse de l'échantillon moyen de ce lait; le poids du beurre obtenu peut nous être très exactement fourni par le beurrier, soit à la sortie de la baratte ou, ce qui vaut mieux, soit après manipulation et conditionnement de celui-ci.

Ce beurre obtenu est l'ultime résultat de l'ensemble de la fabrication, au cours de laquelle toutes les pertes possibles ont pu se produire, pertes inévitables et pertes évitables. Nous a vons done raison de dire que cet overrun réel était bien l'image parfaite du travail tel qu'il s'est effectué.

Quel va être l'enseignement qu'il pourra fournir à l'industriel?

Si l'overrun théorique repose.sur le beurre qu'on aurait dû obtenir et l'overruu réel sur le beurre qu'on a obtenu, toute différence entre ces deux chiffres représentera un déficit de fabrication.

Pour calculer l'overrun théorique, on a tenu compte des pertes en matière grasse inévitables par l'écrémage et le barattage (pertes dans le lait écrémé et le babeurre), pertes qui sont à l'avance connues et strictement dosées par l'analyse de ces sous-produits.

Pour calculer l'overrun réel, on fait abstraction de toutes ces données et on s'adresse brutalement au poids de beurre sorti de la baratte.

En principe done, dans une fabrication parfaite, même en tenant compte des pertes par le lait écrémé et le babeurre, les deux overruns doivent être égaux. Et cependant ils ne le sont jamais. Nous ne pouvons pas incriminer ici l'humidité du beurre, ou son coefficient de pureté, puisque ce coefficient agit de la même manière sur le calcul de l'un et de l'autre; il faut done chercher ailleurs les causes de cette différence. 


\section{DISCUSSION DE LA DIFFÉRENCE ENTRE LES DEUX OVERRUNS.}

Si nous nous reportons au chapitre IV qui traite des pertes évitables et inévitables, nous consta tons que, dans toute usine, quelle qu'elle soit, certaines pertes sont indépendantes de la volonté du personnel et doivent nécessairement exister (nous ne parlons pas bien entendu ici des pertes chiffrées dans le lait écrémé et le babeurre). Nous voyons également que d'autres se produisent fréquemment, mais peuvent la plupart du temps ne pas sè renouveler avec un peu de soin et de surveillance: ce sont celles que nous avons qualifiées "évitables". L'ensemble de ces pertes non chiffrées constitue ce que nous appelons les "pertes indéterminées ».

C'est alors que nous disons que la différence entre les deux overruns est l'image rigoureuse de ces pertes indéterminées, cette différence est évidemment exprimée en $\%$ de la matière grasse entrée.

Prenons quelques exemples dans le tableau de rendement que nous avons reproduit plus haut.

Dans la journée du 3 décembre, la matière grasse entrée était de $147 \mathrm{k} .200$. Les pertes inévitables chiffrées par le lait écrémé ( $3 \mathrm{k} .100$ ) et par le babeurre ( 0 k. 260) forment un total de 3 k. 360 .

La matière grasse qu'on aurait dû obtenir dans le beurre après barattage (s'il n'y avait pas eu d'autres pertes) était done de :

$$
147,200-3,360=143 \mathrm{k} .840 .
$$

L'humidité du beurre obtenu étant de $16 \%$, son coefficient de pureté de $82 \%$, ces 143 k. 840 devaient donner :

$$
\frac{143,840 \times 100}{82}=175 \mathrm{k} .400 \text { de beurre. }
$$

L'overrun théorique est done :

$$
\frac{(175,400-147,200) \times 100}{147,200}=19,16 \% \text {. }
$$

Mais au barattage nous n'avons obtenu que $175 \mathrm{k}$. de beurre, donc nous enregistrons une perte indéterminée de :

$$
175,400-175=0 \mathrm{k} .400 \text { de beurre }
$$

et l'overrun réel est :

$$
\frac{(175-147,200) \times 100}{147,200}=18,89 \% .
$$

La différence entre les deux overruns se chiffre donc par :

$$
19,16-18,89=0,27 \% \text {. }
$$


Nous a vons dit, il y a un instant, qu'une corrélation absolue existait entre cette différence et les pertes indéterminées ; en effet:

Si nous rapportons à 100 de la matière grasse entrée la perte indéterminée en beurre trouvée ci-dessus, nous obtenons :

$$
\frac{0,400 \times 100}{147,2}=0,27 \% \text {, }
$$

chiffre identique à la différence entre les deux overruns.

Nous pouvons, en passant, conclure que le travail de cette journée a été très satisfaisant, puisque les pertes indéterminées en beurre (et, par ricochet, la différence entre les overruns) sont très faibles. L'attention du Directeur ne peut être attirée que par le dosage de la matière grasse dans le lait écrémé qui affirme un défaut léger d'écrémage.

Si nous examinons par contre la journée du 9 décembre, nous constatons, en plus du taux un peu élevé de matière grasse perdue dans le lait écrémé $(0,75$ par litre), une différence importante entre les deux overruns $(4,03 \%)$ et une perte indéterminée en beurre également élevée ( $6 \mathrm{k}, 600$ ). - C'est ici que les notes de fabrication vont nous être d'un précieux secours, car il s'est produit certainement quelque chose d'anormal.

En effet, nous relevons ces indications :

a) perte de crème, engorgement du réfrigérant à crème ;

b) retard dans le nettoyage du bol.

Ceci veut dire que, d'une part, la mise en route de l'agitateur du pasteurisateur à crème a été faite prématurément, a produit un commencement de barattage dans cet a ppareil et les grumeaux de beurre formés ont bouché les trous de la nochère supérieure du réfrigérant, occasionnant un débordement de la crème ; et d'autre part, que le bol, plein de boues, et non changé à temps, a causé momentanément un mauvais écrémage sensible à l'analyse de l'échantillon moyen de lait écrémé.

Nous relevons dans le même ordre d'idée au ler décembre, où la différence entre les overruns est de $1,86 \%$ et les pertes indéterminées de $2 \mathrm{k}$. 500 , la chute de la courroie du réchauffeur à lait avant écrémage. Ce réchauffeur a débordé et une certaine quantité de lait s'est perdue sur le sol.

Nous constatons, pour terminer, que le taux d'humiditédu beurre n’influence pas la différence entre les overruns. Cela se conçoit aisément :

Prenons le cas de la journée du 15 où les notes de fabrication nous révèlent un barattage long et pénible a vee délaitage défectueux. Le taux d'humidité du beurre est de $18 \%$. Par contre, nous voyons que 
la différence entre les overruns n'est que de $0,50 \%$ : l'humidité excessive du beurre n'a donc eu aucune influence sur cette différence, en effet :

Pour ealculer l'overrun théorique, on trouve le poids du beurre qu'on aurait dû obtenir en divisant la quantité de matière grasse qu'on aurait dû avoir par le coefficient de pureté qui lui-même est fonction de l'humidité, en un mot ce beurre qu'on aurait dû obtenir est calculé comme s'il était à $18 \%$ d'humidité. De même, l'overrun réel est obtenu à l'aide du poids du beurre réellement fabriqué qui est lui-même à $18 \%$ d'humidité, donc la différence n'est pas influencé par ce taux d'humidité.

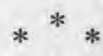

\section{CONCLUSION.}

Toute cette étude aboutit à la constatation bien nette qu'il faut à tout prix, dans une exploitation beurrière, tenir un grand compte du rendement si l'on veut obtenir une fabrication soignée. Le calcul de ce rendement est chose relativement simple et les directives que nous a vons essa yé de donner pour l'effectuer fournissent, à notre a vis, l'ensemble des constatations qui sont nécessaires et suffisantes aux Directeurs de ces industries pour en connaître le bon fonctionnement.

Les analyses renseignent sur la nature du produit entré sur sa qualité et sur ce qu'il doit rendre; elles indiquent avec certitude la perfection de l'écrémage et du barattage ; elles font connaître la qualité, la valeur marchande et la pureté du beurre.

Les mesurages et pesées fournissent des données statistiques très utiles sur la marche générale de l'affaire.

Les calculs enfin permettent, à coup sûr, de savoir si la matière première transformée corres pond bien à la matière première reçue, si l'extraction des principes utiles de cette dernière a été effectuée avec le maximum de soins et le minimum de pertes.

Les commentaires et autres constatations ainsi que les conseils parfois un peu trop détaillés que nous a vons donnés suffiront à conduire les industriels beurriers vers une compréhension sûre du travail qu'ils font exécuter et à les aider dans la réalisation d'une technique rationnelle et raisonnée de leur fabrication.

Nous n'avons voulu faire aucune innovation, mais avons seulement cru être utile en commentant et en ada ptant à notre industrie le beau travail d'HunziKer pour le rendre accessible à tous. 